\title{
STABILITY AND SKEW-PRODUCT FLOWS
}

\author{
JIRŌ EGAWA
}

(Communicated by George R. Sell)

\begin{abstract}
We consider a skew-product flow with a minimal flow as the basic flow, and we show that, if a bounded solution is positively uniformly stable, then the $\omega$-limit set of it is a minimal set. This is an extension of a result in [2], where it was assumed that the basic flow is equicontinuous.
\end{abstract}

Let $R$ denote the set of real numbers and $R^{n}$ the Euclidean $n$-space with the usual norm $|\cdot|$. Let $X$ be a metric space with the metric $d_{X}$. A continuous mapping $\pi: X \times R \rightarrow X$ is called a flow on (a phase space) $X$ if $\pi$ satisfies the following two conditions:

(1) $\pi(x, 0)=x$ for $x \in X$.

(2) $\pi(\pi(x, t), s)=\pi(x, t+s)$ for $x \in X$ and $t, s \in R$.

The orbit of $\pi$ through $x \in X$ is denoted by $C_{\pi}(x)=\{\pi(x, t) ; t \in R\}$. The closure of a set $A \subset X$ is denoted by $\bar{A}$. The $\omega$-limit set of $x \in X$ is denoted by $\Omega^{+}(x)=\left\{y \in X ; \pi\left(x, t_{n}\right) \rightarrow y\right.$ for some sequence $\left\{t_{n}\right\} \subset R$ such that $t_{n} \rightarrow \infty$ as $n \rightarrow \infty\}$. A nonempty set $M \subset X$ is called an invariant set of $\pi$ if we have $C_{\pi}(x) \subset M$ for every $x \in M$. The restriction of $\pi$ to an invariant set $M$ is denoted by $\pi \mid M$. A nonempty compact set $M \subset X$ is called a minimal set of $\pi$ if we have $\overline{C_{\pi}(x)}=M$ for each $x \in M$. If $X$ is itself a minimal set of $\pi$, we say that $\pi$ is a minimal flow on $X$. Let $\pi_{1}$ and $\pi_{2}$ be flows on $X_{1}$ and $X_{2}$, respectively. A continuous mapping $h$ from $X_{1}$ onto $X_{2}$ is called a homomorphism from $\pi_{1}$ to $\pi_{2}$ if we have $h\left(\pi_{1}\left(x_{1}, t\right)\right)=\pi_{2}\left(h\left(x_{1}\right), t\right)$ for $\left(x_{1}, t\right) \in X_{1} \times R$. A homomorphism $h$ from $\pi_{1}$ to $\pi_{2}$ is said to be of negatively distal type if for each $x_{2} \in X_{2}$ and for a pair of distinct points $x_{1}^{\prime}, x_{1}^{\prime \prime} \in h^{-1}\left(x_{2}\right)$ we have

$$
\inf _{t \leq 0}\left\{d_{X_{1}}\left(\pi_{1}\left(x_{1}^{\prime}, t\right), \pi_{1}\left(x_{1}^{\prime \prime}, t\right)\right)\right\}>0 .
$$

If further $\inf _{t \in R}\left\{d_{X_{1}}\left(\pi_{1}\left(x_{1}^{\prime}, t\right), \pi_{1}\left(x_{1}^{\prime \prime}, t\right)\right)\right\}>0$, we say that $h$ is of distal type. Let $\sigma$ be a flow on $X$. We consider a skew-product flow $\pi=(\sigma, \phi)$ on $X \times R^{n}$, that is,

$$
\pi((x, y), t)=(\sigma(x, t), \phi(x, y, t))
$$

for $(x, y) \in X \times R^{n}$ and $t \in R . \pi=(\sigma, \phi)$ become a flow on $X \times R^{n}$ if and only if $\phi$ satisfies the following two conditions.

(1) $\phi(x, y, 0)=y$ for $(x, y) \in X \times R^{n}$.

(2) $\phi(x, y, t+s)=\phi(\sigma(x, t), \phi(x, y, t), s)$ for $(x, y) \in X \times R^{n}$ and $t, s \in R$.

We say that $\sigma$ is the basic flow of $\pi$, and that $\phi(x, y, t)$ is the solution through $(x, y)$.

Received by the editors January 24, 1986 and, in revised form, March 24, 1986.

1980 Mathematics Subject Classification (1985 Revision). Primary 34C35, 34C28; Secondary $54 \mathrm{H} 20$.

Key words and phrases. Skew-product flow, uniformly stable, minimal set, minimal flow, $\omega$-limit set, of distal type, homomorphism. 
A solution $\phi(x, y, t)$ through $(x, y)$ is said to be positively uniformly stable if for each $\varepsilon>0$ there exists a $\delta>0$ such that

$$
\left|\phi(x, y, \tau+t)-\phi\left(\sigma(x, \tau), y^{\prime}, t\right)\right|<\varepsilon \quad(\text { for } t \geq 0),
$$

whenever $\tau \in R$ and $\left|\phi(x, y, \tau)-y^{\prime}\right|<\delta$.

LEMMA 1. Let $\sigma$ be a minimal flow on a compact metric space $X$, and $\pi=$ $(\sigma, \phi)$ a skew-product flow on $X \times R^{n}$. If a bounded solution $\phi(x, y, t)$ through $(x, y) \in X \times R^{n}$ is positively uniformly stable, then for each $\varepsilon>0$ there exists a $\delta(\varepsilon)>0$ such that for each $\left(x^{*}, y^{*}\right) \in \Omega^{+}(x, y)$ we have

$$
\left|\phi\left(x^{*}, y^{*}, \tau+t\right)-\phi\left(\sigma\left(x^{*}, \tau\right), y^{\prime}, t\right)\right|<\varepsilon \quad(\text { for } t \geq 0),
$$

whenever $\tau \in R$ and $\left|\phi\left(x^{*}, y^{*}, \tau\right)-y^{\prime}\right|<\delta$. We say that the function $\delta(\varepsilon)$ is the modulus of uniform stability for $\phi(x, y, t)$.

ProOF. See Theorem 4 in [2].

Let $X$ be a metric space. The set of compact subsets of $X$ is denoted by $\operatorname{Com}(X)$. For $K \in \operatorname{Com}(X)$, and for $r>0$, we denote the set $\left\{x \in X ; d_{X}(x, K)<r\right\}$ by $V_{r}(K)$. For $K_{1}, K_{2} \in \operatorname{Com}(X)$ we define

$$
\rho\left(K_{1}, K_{2}\right) \in \inf \left\{r ; K_{2} \subset V_{r}\left(K_{1}\right) \text { and } K_{1} \subset V_{r}\left(K_{2}\right)\right\} .
$$

Then $\rho$ is a metric on $\operatorname{Com}(X)$. By this $\rho \operatorname{Com}(X)$ becomes a metric space.

LEMMA 2. Let $X$ and $Z$ be metric spaces, and let $h$ be a mapping from $Z$ into $\operatorname{Com}(X)$. Define a mapping $k$ from $X \times Z$ to $R$ by $k(x, z)=d_{X}(x, h(z))$ for $(x, z) \in X \times Z$. If $h$ is continuous, then $k$ is also continuous.

PROOF. Easy.

THEOREM. Let $\sigma$ be a minimal flow on a compact metric space $X$, and let $\pi=(\sigma, \phi)$ be a skew-product flow on $X \times R^{n}$. If a bounded solution $\phi(x, y, t)$ through $(x, y) \in X \times R^{n}$ is positively uniformly stable, then $\Omega^{+}(x, y)$ is a compact minimal set of $\pi$.

ProOF. Since $X$ is compact and $\phi(x, y, t)$ is bounded for $t \geq 0, \Omega^{+}(x, y)$ is compact. Let $M \subset \Omega^{+}(x, y)$ be a minimal set of $\pi$. We shall prove that $M=$ $\Omega^{+}(x, y)$. We consider the following metric on $X \times R^{n}$ :

$$
d_{X \times R^{n}}\left(\left(x_{1}, y_{1}\right),\left(x_{2}, y_{2}\right)\right)=d_{X}\left(x_{1}, x_{2}\right)+\left|y_{1}-y_{2}\right|
$$

for $\left(x_{1}, y_{1}\right),\left(x_{2}, y_{2}\right) \in X \times R^{n}$. The restriction $P \mid M$ of the projection $P: X \times R^{n} \rightarrow$ $X$ to $M$ is a homomorphism from $\pi \mid M$ to $\sigma$, and it is of negatively distal type [2, p. 19]. Hence it is of distal type [1, p. 102]. Consequently $h: X \rightarrow \operatorname{Com}(M)$ defined by $h(x)=M \cap P^{-1}(x)$ for $x \in X$ is continuous [1, p. 105]. Since $\operatorname{Com}(M) \subset$ $\operatorname{Com}\left(X \times R^{n}\right), h$ is continuous as the mapping from $X$ to $\operatorname{Com}\left(X \times R^{n}\right)$. Put $h(x)=\{x\} \times M_{x}\left(M_{x} \subset R^{n}\right), U_{r}(x)=\left\{y \in R^{n} ; d_{R^{n}}\left(y, M_{x}\right)<r\right\}$ for $r>0$, and $U_{r}=\bigcup_{x \in X}\{x\} \times U_{r}(x)$. Then $U_{r}$ is an open subset of $X \times R^{n}$ which contains $M$. In fact, if $U_{r}$ is not open, then there exist $\left(x_{0}, y_{0}\right) \in U_{r}$ and $\left(x_{n}, y_{n}\right) \notin U_{r}$ $(n=1,2, \ldots)$ such that $\left(x_{n}, y_{n}\right) \rightarrow\left(x_{0}, y_{0}\right)$ as $n \rightarrow \infty$. Since $\left(x_{n}, y_{n}\right) \notin U_{r}$, we have $d_{R^{n}}\left(y_{n}, M_{x_{n}}\right) \geq r$ for $n=1,2, \ldots$ Since

$$
d_{X \times R^{n}}\left(\left(x_{0}, y_{0}\right), h\left(x_{0}\right)\right)=d_{X \times R^{n}}\left(\left(x_{0}, y_{0}\right), x_{0} \times M_{x_{0}}\right)=d_{R^{n}}\left(y_{0}, M_{x_{0}}\right),
$$


we have

$$
\lim _{n \rightarrow \infty} d_{X \times R^{n}}\left(\left(x_{n}, y_{n}\right), h\left(x_{n}\right)\right)=d_{X \times R^{n}}\left(\left(x_{0}, y_{0}\right), h\left(x_{0}\right)\right)=d_{R^{n}}\left(y_{0}, M_{x_{0}}\right) \geq r
$$

by Lemma 2 . This implies that $\left(x_{0}, y_{0}\right) \notin U_{r}$. This is a contradiction. Hence $U_{r}$ is open in $X \times R^{n}$.

Further, it is easy to verify that $\left\{U_{r}\right\}_{r>0}$ is a fundamental system of neighborhoods of $M$. If $\Omega^{+}(x, y)$ is not minimal, then there exists $\left(x_{0}, y_{0}\right) \in \Omega^{+}(x, y)-M$. Next choose a neighborhood $V$ of $M$ such that $\left(x_{0}, y_{0}\right) \notin \bar{V}$. Then there exists an $\varepsilon>0$ such that $U_{\varepsilon} \subset V$. Take a point $\left(x^{\prime}, y^{\prime}\right) \in U_{\delta}$, where $\delta(\varepsilon)>0$ is the modulus of uniform stability for the solution $\phi(x, y, t)$. Choose $\left(x^{\prime}, y^{*}\right) \in M$ such that $\left|y^{\prime}-y^{*}\right|<\delta$. Then, by Lemma 1 , we have $\left|\phi\left(x^{\prime}, y^{*}, t\right)-\phi\left(x^{\prime}, y^{\prime}, t\right)\right|<\varepsilon$ for $t \geq 0$. Since $M$ is an invariant set of $\pi$, we have $\left(\sigma\left(x^{\prime}, t\right), \phi\left(x^{\prime}, y^{*}, t\right)\right) \in M$ for $t \geq 0$, and hence $\phi\left(x^{\prime}, y^{\prime}, t\right) \in U_{\varepsilon}\left(\sigma\left(x^{\prime}, t\right)\right)$ for $t \geq 0$. This implies that $\left(\sigma\left(x^{\prime}, t\right), \phi\left(x^{\prime}, y^{\prime}, t\right)\right)=\pi\left(\left(x^{\prime}, y^{\prime}\right), t\right) \in U_{\varepsilon}$ for $t \geq 0$. Since $M \subset \Omega^{+}(x, y)$ and $U_{\delta}$ is an open neighborhood of $M$, there exists $\tau>0$ such that $\pi((x, y), \tau) \in U_{\delta}$. Hence, by the above, $\pi(\pi((x, y), \tau), t)=\pi((x, y), \tau+t) \in U_{\varepsilon}$ for $t \geq 0$. This implies that $\Omega^{+}(x, y) \subset \bar{U}_{\varepsilon} \subset \bar{V}$. Since $\left(x_{0}, y_{0}\right) \in \Omega^{+}(x, y)$ and $\left(x_{0}, y_{0}\right) \notin \bar{V}$, this is a contradiction. Hence we can conclude $\Omega^{+}(x, y)=M$, and hence $\Omega^{+}(x, y)$ is a minimal set of $\pi$.

REMARK 1. In [2] R. J. Sacker and G. R. Sell showed that if $\sigma$ is equicontinuous in the Theorem, then $\Omega^{+}(x, y)$ is a distal minimal set of $\pi$. The Theorem is an extension of this result.

REMARK 2. Let $C\left(R, R^{n}\right)$ be the set of continuous functions from $R$ to $R^{n}$ with the compact-open topology. Define a flow $\eta$ on $C\left(R, R^{n}\right)$ by $\eta(f, t)=f_{t}$ for $(f, t) \in C\left(R, R^{n}\right) \times R$, where $f_{t}(s)=f(t+s)$ for $s \in R$. We denote the hull of $f,{\overline{\left\{f_{t}\right\}}}_{t \in R}$, by $H(f)$. We say that $f$ is a recurrent function if $H(f)$ is a compact minimal set of $\eta$. We can easily show that for every $\left(x^{*}, y^{*}\right) \in \Omega^{+}(x, y)$ in the Theorem, $\phi\left(x^{*}, y^{*}, \cdot\right)$ is a recurrent function.

REMARK 3 . Let $W$ be an open subset of $R^{n}$, and let $C\left(W \times R, R^{n}\right)$ be the set of continuous functions from $W \times R$ to $R^{n}$ with the compact-open topology. Define a flow $\xi$ on $C\left(W \times R, R^{n}\right)$ by $\xi(f, t)=f_{t}$ for $(f, t) \in C\left(W \times R, R^{n}\right) \times R$, where $f_{t}(x, s)=f(x, t+s)$ for $(x, s) \in W \times R$. We denote the hull of $f,{\overline{\left\{f_{t}\right\}}}_{t \in R}$, by $H_{\xi}(f)$. We say that, if $H_{\xi}(f)$ is a compact minimal set of $\xi, f$ is a recurrent function. Let $f$ be a recurrent function. We assume that for $g \in H_{\xi}(f)$ and $x_{0} \in W$ there is a unique solution $\phi\left(g, x_{0}, t\right)$ of the initial valued problem

$$
x^{\prime}=g(x, t), \quad \phi\left(g, x_{0}, 0\right)=x_{0}
$$

defined on $R$. Then $\pi=\left(\xi \mid H_{\xi}(f), \phi\right)$ is a skew-product flow which satisfies the conditions in the Theorem.

\section{REFERENCES}

1. B. M. Levitan and V. V. Zhikov, Almost periodic functions and differential equations, Cambridge Univ., Cambridge, 1982. (English translation)

2. R. J. Sacker and G. R. Sell, Lifting properties in skew-product flows with applications to differential equations, Mem. Amer. Math. Soc. No. 190 (1977).

Department of Mathematics, College of liberal Arts, Kobe University, NADA KOBE 657, JAPAN 